\title{
INVESTIGACIÓN E INNOVACIÓN EDUCATIVA DESDE EL MÁSTER DE FORMACIÓN DEL PROFESORADO DE CIENCIAS SOCIALES A LAS PRÁCTICAS CURRICULARES EN EDUCACIÓN SECUNDARIA
}

\author{
Laura Triviño Cabrera \\ Cristian Requena Palacios \\ Universidad de Málaga
}

\begin{abstract}
RESUMEN: El presente artículo persigue mostrar cómo profesora y tutora universitaria y alumno en prácticas del Máster Universitario de Profesorado en ESO y Bachillerato, en la especialidad de Ciencias Sociales, de la Universidad de Málaga, desarrollaron paralelamente trabajos por proyectos cuya finalidad era la innovación educativa desde la coeducación audiovisual del alumnado del MAES y del curso de $2^{\circ}$ ESO del IES Huerta Alta (Alhaurín de la Torre). Mientras la profesora tutora aplicaba un diseño de investigación educativa en las asignaturas Diseño y Desarrollo de Programaciones y Actividades Formativas y El Currículum de Historia, Geografía y Filosofía obligatorias del MAES que tenía como meta la alfabetización mediática e informacional del alumnado, centrándose en la de/construcción de los videoclips; el alumno en prácticas, implementaba un similar diseño en la asignatura Cambios sociales y Género, partiendo de la televisión. Los resultados de las producciones audiovisuales, tanto del alumnado del máster como del alumnado de ESO, fueron presentados y difundidos en las II Jornadas Clara Peeters y sus otras historias: ¡Empodérate Audiovisualmente!, celebradas en la Facultad de Ciencias de la Educación de la Universidad de Málaga.
\end{abstract}

PALABRAS CLAVE: aprendizaje-servicio, trabajo por proyectos, coeducación audiovisual, currículum oculto, formación del profesorado.

\section{EDUCATIONAL RESEARCH AND INNOVATION FROM MASTER'S CLASSROOM IN SOCIAL STUDIES TEACHER TRAINING TO CURRICULAR PRACTICES IN SECONDARY EDUCATION'S CLASSROOM}

\footnotetext{
ABSTRACT: This article tries to explain how professor-academic tutor and trainee student from Master in Secondary Teacher Training, specialize in
} 
Social Sciences, of the Málaga University, developped in parallel projectsbased learning. The aim of the projects was the audiovisual co-education of students of that master and students at the Huerta Alta High School. While professor-academic tutor was applying an educational research design on Media and Information Literacy in two subjects (Design and development of lessons and classroom activities and History, Geography and Philosophy Curriculum); the trainee student was applying a similar educational research design in the subject: Social Changes and Gender. The results of these projects were the creation of audiovisual productions. Students of the master made videoclips and students of the high school made a short film. All audiovisual productions were introduced by students in Clara Peeters Seminar: Audiovisual Empowerment!

KEYWORDS: service-learning, project-based instruction, audiovisual coeducation, hidden curriculum, teacher training.

Recibido: 01/01/2018

Aceptado: 28/08/2018

Correspondencia: Laura Triviño Cabrera, Universidad de Málaga, Avda. Cervantes, 2, 29071 Málaga. Email: laura.trivino@uma.es.

\section{INTRODUCCIÓN}

El presente trabajo persigue dar a conocer un proyecto aprendizaje-servicio cuyo objetivo principal es la coeducación audiovisual en segundo curso de educación secundaria del IES Huerta Alta de Alhaurín de la Torre (Málaga), en el transcurso de las prácticas profesionales del Máster Universitario del Profesorado en ESO y Bachillerato, en la especialidad de Ciencias Sociales, de la Universidad de Málaga (en adelante, MAES) durante el curso 2016/2017. El proyecto titulado "Televisión en la Escuela, Alfabetización mediática y Cuestiones de género: Iguales ante la cámara, iguales ante el mundo" fue implementado en Cambios sociales y género, asignatura optativa de segundo de educación secundaria.

La principal novedad de estas prácticas profesionales, donde fue desarrollado dicho proyecto, reside en que se empezó a forjar al mismo tiempo que el alumno asistía a las clases de las asignaturas Diseño y Desarrollo de Programaciones y Actividades Formativas y El Currículum de Historia, Geografía y Filosofía del MAES, donde se desarrollaba un trabajo por proyectos que tenía como principal objetivo, la alfabetización mediática del alumnado y el desvelamiento del currículum oculto en cuanto a la invisibilidad de las mujeres en los libros de texto. Así pues, la idea del aprendizaje-servicio empieza a forjarse cuando el alumno decidió, por iniciativa propia, aplicar el mismo proceso enseñanza-aprendizaje del MAES en el segundo curso de educación secundaria del centro escolar donde estaba realizando sus prácticas profesionales. 
Por tanto, se iniciaba la aplicación de una metodología aprendizaje-servicio. El alumno del MAES se alfabetizaba mediáticamente y conocía una pedagogía feminista; para posteriormente, proceder a la alfabetización mediática de sus estudiantes y la toma de una conciencia feminista en la asignatura Cambio social y género en educación secundaria. He aquí la primera parte de la metodología, "aprendizaje". En cuanto al segundo proceso, el servicio a la comunidad, en ambos proyectos tuvo lugar, la producción audiovisual por parte del alumnado con el objetivo de desarrollar el pensamiento crítico en receptores/as audiovisuales; consiguiendo traspasar el aprendizaje de un aula a la ciudadanía. Tanto los trabajos audiovisuales del alumnado del MAES como del alumnado de ESO fueron presentados públicamente en las jornadas de formación "Clara Peeters y sus otras historias: ¡Empodérate audiovisualmente!”, organizadas del 27 al 29 de marzo de 2017 en la Facultad de Ciencias de la Educación de la Universidad de Málaga. En dichas jornadas, asistieron estudiantes desde la ESO hasta estudiantes del Grado en Maestra/o en Educación Primaria.

Así pues, el presente trabajo aborda cómo alumno del MAES y profesora universitaria del MAES y tutora académica de dicho alumno, a través de las prácticas profesionales, consiguieron que la innovación educativa pasara del aula universitaria al aula de educación secundaria. A lo largo del texto, presentaremos cómo fuimos desarrollando y aplicando nuestros diseños de investigación que, en todo momento, conectaban entre sí.

\section{Coeducación audiovisual para un Currículum postmoderno}

\subsection{Del currículum invisible al currículum postmoderno. La perspectiva de género y la cultura mediática en el MAES}

Como profesora del MAES, impartir las asignaturas Diseño y Desarrollo de Programaciones y Actividades Formativas y El Currículum de Historia, Geografía y Filosofía del MAES durante los meses de enero, febrero y marzo de 2017, nos brindó la oportunidad de proceder a la formación del profesorado de Ciencias Sociales en la coeducación audiovisual, entendiendo por ésta:

El necesario reconocimiento de la crucial importancia de una perspectiva de género para el análisis de las imágenes, que se nutra de las plurales y fértiles tradiciones de la teoría feminista y los estudios de género para acometer la lectura crítica de los textos audiovisuales nos permite ir aún más allá al defender una auténtica co-educación audiovisual, cuyo interés simultáneo por la divulgación de la recepción crítica, tanto de la imagen artística como de la mediática, además aspire a lo que hemos Ilamado [...] «coeducar la mirada». (Belmonte, 2014, pp. 150-151)

La coeducación audiovisual nos permitió ahondar en competencias generales y básicas de dichas asignaturas como:

- Buscar, obtener, procesar y comunicar información (oral, impresa, audiovisual, digital o multimedia), transformarla en conocimiento y aplicarla en los 
procesos de enseñanza y aprendizaje en las materias propias de la especialización cursada.

- Diseñar y desarrollar espacios de aprendizaje con especial atención a la equidad, la educación emocional y en valores, la igualdad de derechos y oportunidades entre hombres y mujeres, la formación ciudadana y el respeto de los derechos humanos que faciliten la vida en sociedad, la toma de decisiones y la construcción de un futuro sostenible.

- Fomentar el espíritu crítico, reflexivo y emprendedor.

- Integrar la formación en comunicación audiovisual y multimedia en el proceso de enseñanza-aprendizaje. (Guía docente de las asignaturas, 2017)

"Coeducar la mirada" del alumnado del MAES nos llevó a un objetivo fundamental: incorporar temas controversiales como la cultura mediática y la perspectiva feminista al currículum escolar. Entendiendo por temas controversiales, "controversial issues" (Hess, 2004, p. 6), temas que suscitan división de opinions dependiendo de la sociedad en la que nos encontremos (Kerr y Huddleston, 2016). El hecho es, que el feminismo es un tema controversial evitado en clase, hasta tal punto que, autoras como Arnot, explican cómo "se ha marginalizado el estudio de la educación de género en las discusiones actuales del ámbito de la educación para la ciudadanía" (2009, p. 20); y McRobbie (2004, p. 257) habla de una "distance from feminism" por parte de las jóvenes. Probablemente, esta circunstancia está muy relacionada con un profesorado que participa del denominado "currículum nulo" o "currículum ausente", "lo que aprendemos de lo que no nos enseñan" (Acaso, 2009, p. 59):

Lo que el docente obvia está íntimamente ligado con su posicionamiento político, seguramente agazapado en lo más profundo de su inconsciente. No incluir nociones básicas de educación sexual, [...], que las chicas no practiquen fútbol. El término [...] es no, porque los profesores ejercen su docencia basándose más en lo que no nos enseñarán (pero luego irreparablemente nos enseñan) que en lo que realmente nos acaban mostrando en sus clases. (Acaso, 2009, p. 59)

Como bien indica Nieves Blanco (2009),

Hablamos de currículo para hacer referencia al contenido de la transmisión cultural, a la selección de la cultura que vamos a poner a disposición de las alumnas y de los alumnos. Y al modo en que la vamos a presentar, concretando nuestra tarea mediadora: con el conocimiento que enseñamos y con las alumnas y los alumnos. (p. 12). [...] es necesario insistir en que cuando utilizamos un libro de texto u otro material, no estamos simplemente enseñando (y aprendiendo) Matemáticas, Historia, Lengua o Música; estamos enseñando (y aprendiendo) cómo es el mundo, cómo ha llegado a ser así, quién ha contribuido a ello y de qué manera, qué vale la pena y qué es insignificante... Y también enseñamos (y aprendemos) quiénes somos nosotros y nosotras, qué se espera que hagamos, qué es posible y qué es apropiado, [...] sucede que la mayoría de los libros escolares reflejan y transmiten los modelos sociales dominan- 
tes, contribuyendo a reproducir sesgos, estereotipos y prejuicios sexistas, clasistas y racistas. (p. 13)

Es por lo que promover un currículum postmoderno, que visibilizase las cuestiones de género era una de las metas en la formación del profesorado en ESO y BachiIlerato de la especialidad de Ciencias Sociales. Así pues, los temas contemplados en las asignaturas estaban relacionados con la introducción de los estudios culturales y feministas en el currículum escolar. ¿Cómo programar propuestas didácticas y actividades desde un currículum postmoderno?

\subsection{Cambios sociales y género en el currículum escolar}

Como alumno en prácticas en el IES Huerta Alta (Alhaurín de la Torre, Málaga), tuvimos la oportunidad de elegir la asignatura y curso donde queríamos desarrollar nuestra propuesta de intervención. El período de prácticas se desarrolló durante los meses de marzo y abril; acudiendo al centro durante el mes de marzo, jueves y viernes; y a las asignaturas del MAES, lunes, martes y miércoles. Dado que, en las asignaturas Diseño y Desarrollo de Programaciones y Actividades Formativas y El Currículum de Historia, Geografía y Filosofía, estábamos abordando la coeducación audiovisual; procedimos a solicitar a nuestro tutor profesional del centro, la implementación de nuestra propuesta didáctica en segundo curso de ESO, donde se estaba impartiendo la asignatura optativa. El principio fundamental de partida era "coeducar la mirada" del alumnado de ESO. A continuación, presentamos nuestras impresiones sobre el proceso enseñanza-aprendizaje que expuse en la ficha de evaluación de las asignaturas del MAES y que fueron esenciales para emprender el proyecto de intervención en el aula de educación secundaria:

Lo que más me ha gustado ha sido el hecho de trabajar con un recurso audiovisual en potencia como es el videoclip. Además, creo que el haber tratado de esta manera tan atractiva las cuestiones de género y el empoderamiento de la mujer ha hecho que me haya concienciado más si cabe sobre este tema y que mi compromiso respecto a éste haya crecido desmesuradamente. Esto no sólo me ha permitido crecer como persona, sino también como docente, ya que siento la necesidad y considero un deber el tratar este tipo de temas transversales en las aulas del presente, para así tener una mejor sociedad en el futuro. (Ficha de Evaluación, 2017)

Para ello, quisimos profundizar en dos de los objetivos de la asignatura recogidos en la Orden de 14 de julio de 2016: "integrar la contribución de las mujeres al patrimonio cultural y científico como protagonistas individuales y de grupo en el conocimiento del pasado" (BOJA $n^{\circ} 144,2016$, p. 338) y "analizar y reflexionar sobre los modelos culturales dominantes para reconocer los principales obstáculos que impiden la igualdad" (BOJA n 144, 2016, p. 338). Se trata de una asignatura que puede ser cursada en los tres primeros cursos de la Educación Secundaria Obligatoria en la comunidad autónoma andaluza. Nuestro proyecto de intervención abordaría los siguientes bloques temáticos: 
- El sistema sexo-género y la construcción social de las identidades masculina y femenina. Transmisión de estereotipos y prejuicios sexistas a través de los agentes de socialización: medios de comunicación social. Análisis del lenguaje escrito, oral y audiovisual. (BOJA n 144, 2016, p. 340)

- Análisis de la desigualdad y situaciones de discriminación de las mujeres. Situación social actual de desigualdad entre hombres y mujeres. División sexual del trabajo y desigual acceso al mundo laboral: trabajo productivo y ámbito público, trabajo reproductivo y ámbito privado. Análisis de la representación de hombres y mujeres en los ámbitos público y privado. Brecha digital de género. La organización social en virtud del sexo en otras culturas y la situación de las mujeres en ellas. Algunas medidas para lograr la igualdad entre los sexos: institucionales, grupales o individuales. (BOJA n 144, 2016, p. 340)

- Visibilización de las mujeres. Aportaciones de las mujeres a los distintos campos del saber y de la cultura. Biografías de mujeres. La visibilización / invisibilización de mujeres y hombres en distintas fuentes de información. El sexismo en el lenguaje: el papel del lenguaje en la representación del mundo, en la regulación de conductas y en la construcción de las identidades masculinas y femeninas. La participación de las mujeres en la cultura y en la historia. La imagen de la mujer en la publicidad, en programas televisivos y en el cine. (BOJA n ${ }^{\circ} 144,2016$, p. 342)

\section{HaCia LA ALFABETIZACIÓN MEDIÁTICA E INFORMACIONAL EN LA EDUCACIÓN FORMAL}

Hasta tal punto es crucial abordar en el plano educativo, el alcance y la influencia de los discursos audiovisuales que la UNESCO puso en marcha en el año 2012 unas pautas para desarrollar la alfabetización audiovisual mediante la publicación del documento denominado Media and Information Literacy. Curriculum for Teachers (traducido al español como Alfabetización Mediática e Informacional. Currículum para Profesores). Con respecto al término Alfabetización Mediática e Informacional (conocido bajo las siglas, AMI), debemos precisar que son dos las escuelas de pensamiento que determinan las clasificaciones de la AMI. Por un lado, aquélla que considera a la alfabetización mediática, caracterizada por enfatizar "la habilidad para entender las funciones de los medios, evaluar cómo se desempeñan aquellas funciones y comprometerse racionalmente con los medios para la auto-expresión" (Wilson, Grizzle, Tuazon, Akyempong y Cheung, 2012, p. 18); integrada en la alfabetización informacional que "enfatiza la importancia del acceso a la información, la evaluación y el uso ético de dicha información" (Wilson, Grizzle, Tuazon, Akyempong y Cheung, 2012, p. 20). Y por otro lado, aquélla establecida por especialistas convocados/as por la UNESCO en la que la Alfabetización Mediática e Informacional deben ir juntas y acaparan el conjunto de los siguientes tipos de alfabetización: mediática, informacional, bibliotecaria, publicitaria, en libertad de expresión, en libertad de 
información, noticiosa, digital, televisiva, computacional, cinematográfica, en internet y en juego.

Y, ¿por qué es tan necesaria la alfabetización mediática e informacional en la formación inicial del profesorado? Para empezar, debemos tener presente que el alumnado universitario es generalmente joven,

formados en una cultura audiovisual y acelerada, con escasa capacidad de concentración, poca práctica de lectura y casi ningún hábito de estudio [...] estudiantes habituados, casi de modo excluyente, a medios y lenguajes audiovisuales, que provienen, en general, de un ambiente en que las formas de pensar están estructuradas a partir de los medios de comunicación [...] estas tecnologías van a establecer un impacto en las formas educativas, similar al que estableció la imprenta en el siglo XV (San Martín, 2004, p. 1).

Por tanto, estamos ante un alumnado que consume cultura audiovisual pero normalmente no reflexiona sobre ésta. Por tanto, la educación debe conseguir infundir a la ciudadanía del entendimiento crítico sobre el "fenómeno social". Así pues, la AMI tiene como objetivo:

empoderar a las personas en todos los ámbitos de la vida para buscar, evaluar, utilizar y crear la información de una forma eficaz para alcanzar sus metas personales, sociales, ocupacionales y educativas. Esto es un derecho básico en un mundo digital y promueve la inclusión social de todas las naciones (Wilson, Grizzle, Tuazon, Akyempong y Cheung, 2012, p.16).

\subsection{El Diseño de Investigación Educativa en el aula del MAES y en el aula de educación secundaria}

El diseño de investigación educativa, tanto en el aula del MAES como en el aula de educación secundaria tuvo dos momentos fundamentales. Por un lado, la deconstrucción crítica de los mensajes audiovisuales desprendidos de la cultura mediática; por otra parte, la construcción de mensajes audiovisuales por parte del alumnado desde una perspectiva de género. Esto es, el paso de receptores/as audiovisuales a emisores/as audiovisuales.

En el caso del MAES, se pasó del análisis crítico del videoclip Formation (2016) de Beyoncé a la realización de cuatro videoclips en grupos de 9/10 estudiantes. Optamos por el uso del videoclip puesto que hoy en día, habría que hablar de la relevancia que ha adquirido el contador de visitas a los videoclips albergados en Youtube. Ante el descenso de ventas de discos, la "visualidad de la música" es el medidor del éxito y de la influencia de los y las artistas pop. Por tanto, en mayor medida, una determinada canción dependerá de su proyección audiovisual, de millones e incluso billones de visualizaciones en Youtube, para llegar a ser un "hit". Hasta tal punto es influyente el videoclip que las letras de las canciones quedan en un segundo plano. Este hecho provoca que cada vez más, las productoras musicales inviertan tanto o más en la realización de los videoclips que en los mensajes 
de las canciones; teniendo como resultado, unas letras de menor calidad, así ha sido reflejado en un reciente estudio encargado por la empresa "Seatsmart" 3 que pretendía medir el grado de inteligencia de 225 canciones que habían conseguido las primeras posiciones en el ranking de Billboard durante diez años. El estudio fija dos interrogantes: ¿No estamos volviendo más tontos? ¿Quién gana la batalla de los sexos?

En segundo curso de la ESO, se procedió al análisis crítico de los roles y los estereotipos de género en la televisión; para que posteriormente, el alumnado, a través del grupo-clase, creara un cortometraje sobre los obstáculos de las niñas para dedicarse al ámbito artístico, teniendo como referente, la visibilidad de la pintora Clara Peeters. Desde hace años expertos/as en materia educativa perciben la necesidad de integrar y enseñar a ver la televisión dentro de los centros educativos (Pérez-Tornero, 1994; Hernández, 1997; Masanet y Ferrés, 2013; Aguaded y Cabero, 2013). Para ello, es de vital importancia que se diseñen proyectos o unidades didácticas en torno a la televisión que permitan al alumnado alfabetizarse mediáticamente -entender, analizar, reflexionar y ser crítico con el paradigma televisivo- y analizar el contenido televisivo desde perspectiva de género, para que así logren localizar, comprender, analizar y reflexionar sobre cómo se trata a hombres y mujeres en la televisión, o si, entre otras cosas, se ponen de manifiesto pensamientos y/o acciones sexistas, machistas, segregadoras, etc.

El objetivo principal de los análisis con perspectiva de género es determinar los mecanismos representativos por los que cualquier texto construye la diferencia sexual de forma jerárquica. Los análisis tienen además una finalidad: destacar qué tienen de opresivos y enajenantes, tanto para hombres como para mujeres, las representaciones que se generan en el orden patriarcal. Una crítica textual con perspectiva de género debe optar por no aceptar las visiones críticas tradicionales y arriesgarse en la búsqueda de nuevos conocimientos y fórmulas interpretativas. (Bernárdez, 2015, p.66)

Es interesante la reflexión de Pérez (2005), quien nos explica el porqué es importante un buen uso de la televisión, no teniendo que evitarla o rehusarla:

La alternativa a la televisión no está en rechazarla o en una pretendida ignorancia de su apabullante presencia, sino en la alfabetización. Esto se traduce necesariamente en comprender los nuevos lenguajes que la televisión incorpora, desentrañar sus estrategias persuasivas, desvelar sus códigos, desmitificar su estatus de referente privilegiado, diferenciar conscientemente imagen y realidad, reconocer manipulaciones y tergiversaciones... Estos objetivos se hallan muy vinculados con las capacidades comunicativas que subyacen tras la competencia mediática, para que la televisión se convierta realmente en un poderoso medio de transformación social y de servicio a los ciudadanos (p.199).

A continuación, presentamos una tabla donde se explica el proceso enseñanzaaprendizaje de ambos proyectos a diferentes niveles: 


\section{Alumnado del MAES}

Alumnado de $2^{\circ} \mathrm{ESO}$

\begin{tabular}{|c|c|c|}
\hline Asignaturas & $\begin{array}{l}\text { Diseño y Desarrollo de Programa- } \\
\text { ciones y Actividades Formativas y } \\
\text { El Currículum de Historia }\end{array}$ & Cambios sociales y género \\
\hline $\begin{array}{l}\text { Número de } \\
\text { estudiantes }\end{array}$ & 44 & 26 \\
\hline Tipo de Trabajo & Trabajo por Proyectos & Trabajo por Proyectos \\
\hline Enfoque & Interdisciplinar & Interdisciplinar \\
\hline $\begin{array}{l}\text { Finalidad } \\
\text { educativa }\end{array}$ & $\begin{array}{l}\text { Alfabetización mediática e infor- } \\
\text { macional }\end{array}$ & $\begin{array}{l}\text { Alfabetización mediática e infor- } \\
\text { macional }\end{array}$ \\
\hline Título del Proyecto & Okay, students, let's get in formation! & $\begin{array}{l}\text { Televisión en la Escuela, Alfabeti- } \\
\text { zación mediática y Cuestiones de } \\
\text { género: Iguales ante la cámara, } \\
\text { iguales ante el mundo }\end{array}$ \\
\hline $\begin{array}{l}\text { Herramienta } \\
\text { educativa para la } \\
\text { AMI }\end{array}$ & $\begin{array}{l}\text { Videoclip Formation (Beyoncé, } \\
\text { 2016) }\end{array}$ & Televisión \\
\hline $\begin{array}{l}\text { Producción } \\
\text { audiovisual del } \\
\text { alumnado }\end{array}$ & $\begin{array}{l}\text { Cuatro videoclips: "Rompiendo } \\
\text { espejos", "Emojis", "Ponte en mi } \\
\text { lugar" y "Que no te digan lo que } \\
\text { tienes que hacer" }\end{array}$ & $\begin{array}{l}\text { Cortometraje: "Pinceladas de Mu- } \\
\text { jer" }\end{array}$ \\
\hline $\begin{array}{l}\text { Creación de } \\
\text { propuestas } \\
\text { didácticas }\end{array}$ & $\begin{array}{l}\text { Talleres didácticos a partir de los } \\
\text { videoclips creados por el propio } \\
\text { alumnado para impartir a estudian- } \\
\text { tes de ESO y Bachillerato durante } \\
\text { las Jornadas }\end{array}$ & $\begin{array}{l}\text { Talleres didácticos sobre televisión } \\
\text { creados por el alumno en prácticas } \\
\text { para forjar el pensamiento crítico } \\
\text { del alumnado de ESO sobre los } \\
\text { programas de la televisión en la } \\
\text { asignatura Cambios sociales y gé- } \\
\text { nero }\end{array}$ \\
\hline $\begin{array}{l}\text { Duración del } \\
\text { proyecto }\end{array}$ & $\begin{array}{l}\text { Tres meses (de enero a marzo, } \\
2017 \text { ) }\end{array}$ & 1 mes (marzo, 2017). \\
\hline $\begin{array}{l}\text { Presentación y } \\
\text { difusión }\end{array}$ & $\begin{array}{l}\text { III Jornadas Clara Peeters y sus otras } \\
\text { historias: ¡Empodérate Audiovisual- } \\
\text { mente! }\end{array}$ & $\begin{array}{l}\text { III Jornadas Clara Peeters y sus otras } \\
\text { historias: ¡Empodérate Audiovisual- } \\
\text { mente! }\end{array}$ \\
\hline $\begin{array}{l}\text { Evaluación del } \\
\text { proyecto }\end{array}$ & $\begin{array}{l}\text { Elaboración de una ficha de eva- } \\
\text { luación con una serie de ítems }\end{array}$ & $\begin{array}{l}\text { Elaboración de una ficha de eva- } \\
\text { luación con una serie de ítems }\end{array}$ \\
\hline Objetivo Educativo & Coeducación Audiovisual & Coeducación Audiovisual \\
\hline
\end{tabular}

Figura 1. Diseño de investigación educativa en el aula del MAES y en el aula de ESO. Fuente: Elaboración propia. 
En lo que se refiere a los momentos de la aplicación del diseño de investigación educativa, variaron significativamente, aunque los pasos esenciales eran similares. Un primer momento en el que se indagó en las ideas previas del alumnado en relación al recurso audiovisual proyectado. En el caso del MAES, se procedió a un comentario individual libre sobre el videoclip para llegar hasta la realización de dos cuestionarios más: por una parte, el cuestionario MELIR (Método para la Lectura de Imágenes que nos Rodean, Acaso, 2009) y una serie de interrogantes propuestas por la UNESCO (Grizzle, Tuazon, Akyempong y Cheung, 2012). En $2^{\circ}$ ESO, se llevó a cabo un cuestionario individual sobre la televisión que había sido elaborado previamente por el alumno en prácticas.

Tras haber ahondado en el conocimiento tácito de las y los discentes, el alumnado del MAES tuvo que plantear talleres didácticos que utilizasen sus propios videoclips como punto de partida para la explicación de contenidos del currículum escolar de ESO y/o Bachillerato de una manera interdisciplinar. Para ello, la profesora proporcionó un dossier con diferentes textos de autoras y autores sobre cultura mediática, estudios culturales y feministas que les ayudasen a preparar tanto los videoclips como los talleres, afrontando la necesidad de promover un currículum postmoderno que contemple las otras historias, la visibilidad de las mujeres y las cuestiones de género, esencialmente.

En cuanto al alumnado de $2^{\circ} \mathrm{ESO}$, el alumno en prácticas desarrolló talleres didácticos sobre la televisión con el objetivo de forjar el pensamiento crítico del alumnado ante los programas de la televisión. Uno de los textos básicos para la elaboración de los talleres fue uno de los textos propuestos en el dossier de las asignaturas del MAES, hablamos del libro Mujeres en Medio (s): Propuestas para analizar la comunicación masiva con perspectiva de género de Asunción Bernárdez Rodal (2015). En dichos talleres se trataron los siguientes aspectos: las características de la televisión como medio de comunicación de masas; el proceso de comunicación que tiene lugar en la televisión; los tipos de lenguaje empleados en televisión; el sistema de calificación por edades de contenidos audiovisuales; los roles televisivos; las funciones de la televisión, haciendo especial hincapié en la influencia como función oculta y centrándonos en aquella influencia que ejerce la televisión en las cuestiones de género, sobre todo en el trato desigual hacia la mujer, trabajando con todo ello la concienciación y la búsqueda de la igualdad y de soluciones en el marco televisivo.

Todos estos contenidos que se han puesto de manifiesto y que han marcado el carácter de los diversos talleres han conformado el proceso de enseñanza-aprendizaje, talleres a través de los cuales se ha intentado que los y las estudiantes adquiriesen los conocimientos y habilidades mencionadas en relación a la televisión. Tras estos talleres, habiendo adoptado el alumnado las competencias críticas y audiovisuales para ello, procedimos a crear nuestro propio programa de televisión, en este caso un cortometraje, pues una película o una serie se antojaban demasiado extensas, todo ello desde un punto de vista crítico y tratando las cuestiones de género ya indicadas.

Finalmente, en cuanto al propio corto, denominado "Pinceladas de Mujer" (Requena, 2017; Requena y Triviño, 2018), se puede decir que en él se ha tratado de 
reflejar el trato que ha recibido la mujer dentro de una sociedad que ha sido y aún sigue respondiendo a la cultura patriarcal, en la que el hombre ha tratado de imponer y conducir el camino que ha habido de recorrer la mujer en este devenir que es la vida, y por consiguiente, en todas las facetas de la misma. En nuestro caso, nos hemos centrado sobre todo en el ámbito laboral, en los roles de género, concretamente, en una profesión destinada a "grandes genios", la creatividad y la genialidad no podían ser alcanzadas por las mujeres. Nos referimos a la profesión de artista. De esta forma, se ha procurado crear un vínculo directo y un paralelismo entre el contenido del corto y la protagonista de las mencionadas jornadas en las que se encuadra el proyecto, la pintora Clara Peeters, ejemplo de artista que alzó sigilosamente el empoderamiento de la mujer pintora, una hazaña que llegará hasta nuestros días cuando el Museo Nacional del Prado en 2016, dedique la primera exposición a una mujer pintora: "El Arte de Clara Peeters". He aquí los títulos y contenidos de los talleres:

- Taller 1: Soy la televisión, ¿de verdad me conoces? En este taller el alumnado trabaja las características de la televisión como medio de comunicación, el proceso de comunicación que se desarrolla en la misma, los tipos de lenguaje que se emplean en la TV, y la simbología de la calificación por edades (2 sesiones completas).

- Taller 2: Y esto, ¿para qué es? En este taller el alumnado trabaja las funciones de la televisión, haciendo hincapié en la función de influenciar y como se produce dicha influencia en las cuestiones relativas al género, insistiendo en las desigualdades y discriminaciones que sufre constantemente la mujer (2 sesiones completas).

- Taller 3: Descubriendo a Clara Peeters. Sesión expositiva para explicar la biografía de Clara Peeters y los principales obstáculos a los que se enfrentó como mujer pintura en su época, así como que fue la primera mujer cuyas obras han tenido una exposición en el Museo del Prado. Finalmente se presentan algunas de las principales obras de la pintora (1 sesión completa).

- Taller 4. ¿Cuál es nuestro rol en la televisión? En este taller el alumnado investiga sobre los principales roles televisivos con el fin de continuar con el proyecto y elaborar sus propios programas de televisión en los que se establezcan patrones de conducta y formas de pensar que favorezcan el empoderamiento de la mujer en pro de la igualdad. 4 sesiones completas.

- Taller 5: Grabación del Cortometraje. En estas sesiones se les entrega a los y las estudiantes el guion el corto y se les explica este paso a paso y lo que vamos a hacer. Seguidamente se asignan los roles o personajes a los diferentes alumnos/as. Se hacen algunas pruebas de grabación para familiarizar al alumnado y se graba el corto (4 sesiones completas).

- Evaluación: Diario de aprendizaje individual y evaluación del profesor en prácticas. En esta sesión el alumnado, individualmente, cumplimenta el diario de aprendizaje individual donde pone de manifiesto todo lo que aprendi- 
do de una manera reflexiva, crítica y personal. Asimismo, evalúa mediante la ficha de evaluación del profesor en prácticas, tanto a éste como el proyecto realizado.

\section{3. ¡EMpodérate Audiovisualmente!: trabajo por proyectos deSDE la METODOLOGÍA APRENDIZAJE-SERVICIO}

Tanto en el MAES como en $2^{\circ}$ ESO, nuestro punto de partida fue el Trabajo por Proyectos (en adelante, TPP). El TPP se muestra como un método de enseñanza que posee un potencial educativo y constructivo, destacando de entre sus características fundamentales: su origen constructivista; su carácter interactivo, colaborativo y cooperativo (Moliner, 2013); la valoración de los procesos sobre el producto, de modo que se valora por encima de todo el progreso del o de la estudiante (Coria, 2011); la importancia que se le otorga a los conocimientos previos de los alumnos y las alumnas, los cuales son el punto de partida del proceso educativo (Pozuelos, 2007); su relación con el método científico (Muñoz y Díaz, 2009), así como con los contextos y/o situaciones reales y cercanas al alumnado (NorthWest Regional Educational Laboratory, 2006); y la consideración del alumno o la alumna como el foco principal del proceso enseñanza-aprendizaje. El objetivo principal es potenciar el desarrollo de conocimientos y de habilidades tales como la reflexión, la capacidad crítica, la investigación o la responsabilidad para con el propio aprendizaje, que no sólo son útiles en el aula, sino también en la vida real.

Asimismo, el TPP fue complementado con la aplicación del aprendizaje-servicio, entendido como

una metodología orientada a la educación para la ciudadanía, inspirada en las pedagogías activas, y compatible con otras estrategias educativas. Es un método para unir éxito educativo y compromiso social: aprender a ser competentes siendo útiles a los demás. [...]. Las prácticas de aprendizaje-servicio se revelan extraordinariamente valiosas, al acercar a los estudiantes al mundo real; al retar su capacidad creativa y emprendedora en resolver problemas por sí mismos y darles confianza en hacerlo; [...]. Un proyecto de aprendizaje-servicio es un proyecto educativo y social al mismo tiempo. [...] (Batllé, 2008, p. 5).

Alumnado del MAES y alumnado de $2^{\circ}$ ESO trabajaron en sus respectivas aulas en un proyecto cuyos fines eran compartidos: la alfabetización mediática e informacional y la perspectiva de género, para llegar a la coeducación de la mirada. La coeducación audiovisual conectaba con el objetivo de las III Jornadas Clara Peeters y sus otras historias: ¡Empodérate Audiovisualmente! En el caso de $2^{\circ} \mathrm{ESO}$, el alumnado se implicó en la visibilidad de las mujeres como sujetos de la historia del arte. De modo que estábamos ante una coeducación en el sentido más amplio de la palabra (Blanco, 1999). 


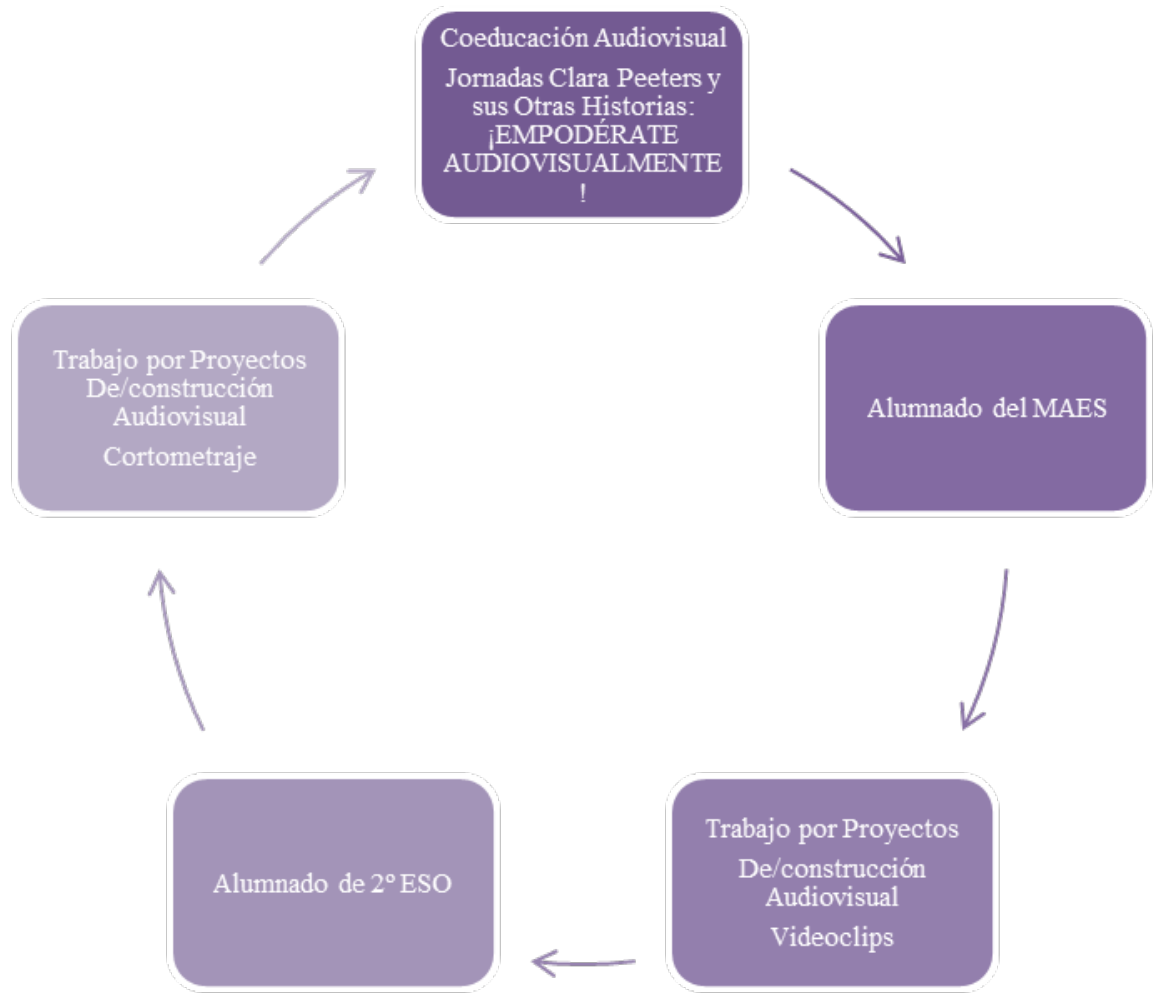

Figura 2. Aprendizaje-Servicio para la Coeducación Audiovisual. Fuente: elaboración propia.

Las dificultades de dichos proyectos fueron fundamentalmente, por una parte, conseguir que el alumnado trabajara en grupo puesto que prima habitualmente el trabajo individual en el ámbito educativo; por otra parte, la necesidad de contar con más sesiones para la planificación de los proyectos. No obstante, el desarrollo de este tipo de trabajos por proyectos, pese a las complejidades, demostró la importancia de que estudiantes consigan trabajar de manera cooperativa y colaborativa. Algunas de las propuestas de mejora de dicho proyecto fueron encaminadas hacia una mejor distribución del tiempo puesto que unos retos tan ambiciosos, como son la elaboración de productos audiovisuales por parte del alumnado, requerían de más sesiones. 


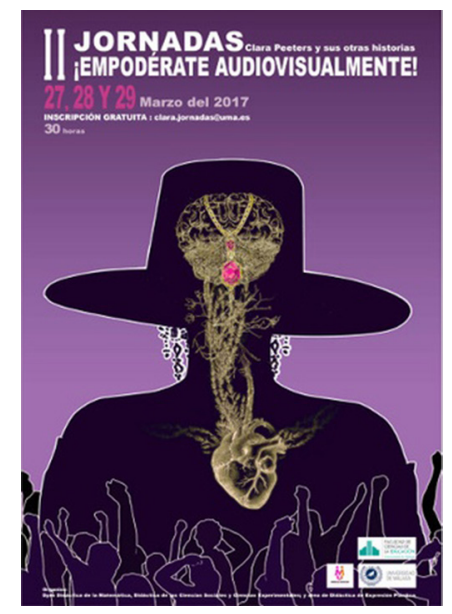

Figura 3. Cartel III Jornadas Clara Peeters y sus otras historias: ¡Empodérate Audiovisualmente!

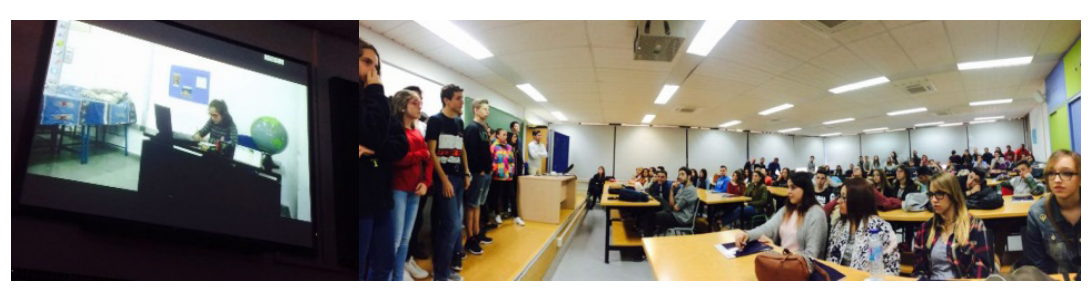

Figuras 4 y 5. Presentación de "Pinceladas de Mujer", IES Huerta Alta. En III Jornadas Clara Peeters y sus otras historias: ¡Empodérate Audiovisualmente! Alumnado del MAES y alumnado de $2^{\circ}$ ESO del IES Huerta Alta. Facultad de Ciencias de la Educación, Universidad de Málaga.

\section{Conclusiones}

Entre las principales conclusiones que extraemos de estas prácticas profesionales, destacamos la importancia de que la tutorización académica y profesional del alumnado del MAES contemple los intereses, las inquietudes, las iniciativas y las motivaciones del alumnado en prácticas. A través de esta experiencia, quisiéramos enfatizar la necesidad de que el profesorado, tanto universitario como de los centros educativos, ofrezcan libertad creativa e innovadora, dentro de las limitadas posibilidades que se les conceden a las prácticas profesionales del máster. Si ambos tutores, tutora de Universidad y tutor del IES Huerta Alta no hubiesen escuchado las propuestas del alumno del MAES, el proyecto Televisión en la Escuela, Alfabetización mediática y Cuestiones de género: Iguales ante la cámara, iguales ante el mundo, no hubiese pasado de una mera ilusión. Pero esa ilusión 
educativa pudo Ilevarse al plano real, hecho que demuestra cómo las prácticas profesionales no son un simple paso insignificante del alumnado del MAES por los centros escolares.

Ahora bien, pese al resultado exitoso de dicho proyecto, del cuestionario de evaluación del profesor en prácticas cumplimentado por sus estudiantes, se deduce la necesidad de que el proyecto hubiese ampliado su duración de un mes a un cuatrimestre, al igual que el proyecto en el MAES, dado la variedad y la complejidad de los temas abordados en los talleres que habían sido tratados por primera vez.

Convertir al alumnado de la ESO en protagonista de un relato audiovisual donde se visibilicen los obstáculos de las mujeres para ejercer determinadas profesiones, como el rol de pintora en este caso y conectar con la biografía de Clara Peeters; conseguir que el alumnado trabaje de forma cooperativa; lograr que el alumnado de ESO presente su cortometraje en unas jornadas universitarias... son momentos que forman parte de un proyecto de innovación educativa que comenzó en un aula del MAES y llegó hasta el aula de educación secundaria, corroborando cómo estamos ante un proyecto aprendizaje-servicio para la coeducación audiovisual,

una forma de aprendizaje activo, integrado en el currículum, en la que los estudiantes aprenden y maduran mediante la participación activa en experiencias de servicio organizadas para adquirir conocimientos y cubrir necesidades sociales. Se trata de una forma de educación experiencial en la que los estudiantes se comprometen en actividades de ayuda a la comunidad al tiempo que facilita el aprendizaje académico y el desarroIlo de competencias profesionales (Rodríguez-Gallego y Ordóñez-Sierra, 2015, p. 315)

Uno de los momentos fundamentales que corroboraron los objetivos fundamentales del proyecto aprendizaje-servicio, fue el intercambio de ideas sobre los relatos audiovisuales propios entre alumnado del MAES y alumnado de ESO. La proyección de los vídeos de los dos grupos de discentes en las jornadas significó que estudiantes de distintos niveles educativos, correspondientes a diferentes generaciones, pudieron entablar un diálogo que propició el desarrollo del pensamiento crítico a través de la proyección de sus producciones audiovisuales para su empoderamiento ciudadano.

\section{REFERENCIAS BIBLIOGRÁFICAS}

Acaso, M. (2009). La educación artística no son manualidades. Nuevas prácticas en la enseñanza de las artes y la cultura visual ( $3^{a}$ ed.). Madrid: Catarata.

Aguaded, J. I. y Cabero, J. (2013). Tecnologías y medios para la educación en la esociedad. Madrid: Alianza.

Arnot, M. (2009). Coeducando para una ciudadanía en igualdad. Madrid: Morata.

Batllé, R. (2008). ¿Qué es el aprendizaje-servicio y por qué nos interesa? Monográficos. Escuela. El aprendizaje-servicio, un método para la EpC y los Derechos Humanos, 4-5. 
Belmonte Arocha, J. (2014). Del arte cinematográfico a la imagen postelevisiva: Coeducación audiovisual ante las representaciones e la feminidad. Dossiers feministes, 19, 149-167.

Bernárdez Rodal, A. (2015). Mujeres en Medio (s): Propuestas para analizar la comunicación masiva con perspectiva de género. Madrid: Fundamentos.

Blanco García, N. (2009). Los saberes de las mujeres y la transmisión cultural en los materiales curriculares. Investigación en la Escuela, 65, 11-22. Recuperado de http://www.investigacionenlaescuela.es/articulos/65/R65_2.pdf.

Blanco García, N. (1999). Coeducación: la apuesta por una pedagogía de sujetos visibles. Kikiriki. Cooperación educativa, 54, 47-52.

Boletín Oficial de la Junta de Andalucía no 145. Orden de 14 de julio de 2016 por la que se desarrolla el currículum correspondiente de bachillerato de la Comunidad Autónoma de Andalucía se regula determinados aspectos de atención a la diversidad y se establece la ordenación de la evaluación del proceso de aprendizaje del alumnado. España, Andalucía, 29 de julio de 2016, 108-396.

Coria Arreola, J. M. (2011). El aprendizaje de proyectos: una metodología diferente. E-formadores, 5. Recuperado de http://red.ilce.edu.mx/sitios/revista/e_ formadores_pri_11/articulos/monica_mar11.pdf.

Hernández Díaz, G. (1997). Aprender a ver la televisión. Comunicar, 8, 111-116. Recuperado de https://www.revistacomunicar.com/verpdf.php?numero=8\&articu lo $=08-1997-16$.

Hess, D. E. (2004). Controversies about Controversial Issues in Democratic Education. PSOnline.

Kerr, D. y Huddleston, T. (Ed.) (2016). Living with Controversy. Teaching Controversial Issues Through Education for Democratic Citizenship and Human Right. Training Pack for Teachers. Council of Europe. Recuperado de http://www.mecd.gob. es/educacion-mecd/dms/mecd/educacion-mecd/mc/convivencia-escolar/ formacion/jornadas-consejo-europa/Teaching-Controversial-issues---professionaldevelopment-pack-for-teache---/Teaching\%20Controversial\%20issues\%20-\%20 professional\%20development\%20pack\%20for\%20teache....pdf.

Masanet Jordà, M. J. y Ferrés Prats, J. (2013). La enseñanza universitaria española en materia de educación mediática. Communication papers: media literacy and gender studies, 2(2), 83-90.

McRobbie, A. (2004). Post-Feminism and Popular Culture. Feminist Media Studies, November, 254-264.

Moliner García, O. (2013). Educación inclusiva. Castellón de la Plana: Publicacions Universitat Jaume I. http://doi.org/10.6035/Sapientia83.

Muñoz, A. y Díaz, M. C. (2009). Metodología por proyectos en el área de conocimiento del medio. Docencia e Investigación: revista de la Escuela Universitaria de Magisterio de Toledo, 34(19), 101-126. Recuperado de http://hdl.handle.net/10578/8158. 
NorthWest Regional Educational Laboratory (2006). Aprendizaje por proyectos. [Traducido al español de Project-Based Instruction: Creating Excitement for Learning]. Colombia: Eduteka.

Pérez Rodríguez, M. A. (2005). El discurso televisivo: un lenguaje seductor que cuenta un mundo virtual. Comunicar, 25, 197-202. Recuperado de https:// www.revistacomunicar.com/index.php?contenido=detalles\&numero $=25 \&$ articu $\mathrm{lo}=25-2005-027$.

Pérez Tornero, J. M. (1994). El desafío educativo de la televisión. Barcelona: Ediciones Paidós Ibérica.

Pozuelos Estrada, F. J. (2007). Trabajo por proyectos: descripción, investigación y experiencias. Morón de la Frontera (Sevilla): Ediciones MCEP.

Requena Palacios, C. (27 de marzo de 2017). Pinceladas de mujer. [Youtube]. Recuperado de https://www.youtube.com/watch?v=vQFsup-zYmA\&t=53s.

Requena Palacios, C. y Triviño Cabrera, L. (2018). El proyecto pinceladas de mujer: enseñar Historia de las mujeres artistas desde la empatía histórica audiovisual. En López Torres, E.; García Ruíz, C. R.; Sánchez Agustí, M. (Eds.), Buscando formas de enseñar: investigar para innovar en Didáctica de las Ciencias Sociales (pp. 781 790). Valladolid: Ediciones Universidad de Valladolid, Asociación Universitaria del Profesorado de Didáctica de las Ciencias Sociales (AUPDCS).

Rodríguez-Gallego, M. R. y Ordóñez-Sierra, R. (2015). Una experiencia de aprendizajeservicio en comunidades de aprendizaje. Revista de currículum y formación del profesorado, 19(1), 315-333. Recuperado de http://www.ugr.es/ recfpro/ rev191ART14.pdf.

San Martín, R. (2004). Primer año. El desafío de dar clase a los recién Ilegados. Diario La Nación, sección 9 Universidades \& Posgrados.

Seatsmart (2015). Lyric Intelligence in Popular Music: A Ten Year Analysis. Recuperado de http://seatsmart.com/blog/lyric-intelligence/. 